Pingping Zhang

Huaiyu $W u$

Lin Ji

Marko Jokić

DOI: $10.21278 /$ TOF.40403

ISSN 1333-1124

eISSN 1849-1391

\title{
A STUDY ON THE EFFECTS OF LOCAL ADDED MASSES ON THE NATURAL AND THE SOUND RADIATION CHARACTERISTICS OF THIN PLATE STRUCTURES
}

\begin{abstract}
Summary
As panel-like structures are widely used in industrial products such as high speed trains, automobiles, and ships, the effects of additional attachments (e.g. lumped mass, rib-stiffeners) to the panels on their dynamic/acoustic characteristics have been investigated analytically, numerically, experimentally or combining two or all the methods in the past decades. The present study focuses on highlighting the differences among local mass effects on the vibration and the radiation behaviour of flexible modes of the flat panel structures. A simple model comprising a local mass attached to a rectangular plate surface is set up, allowing us a deep insight into how the local mass affects the inherent mode parameters and the corresponding vibration and radiation characteristics of panel structures. The influential phenomena are first investigated analytically and then verified using FE-numerical simulations. The results show that: (1) the dynamic modal parameters of flat panel structures show different sensitivity to the values of the added mass and its locations; (2) the vibration and radiation characteristics of elastic modes with the same order can be affected in quite different degrees by the same local mass attachment; and (3) the modal acoustic interactions of thin plates can be significantly affected by the local mass attachments.
\end{abstract}

Key words: $\quad$ Local mass, panel structure, dynamic modal property, radiation characteristics, self-/mutual-radiation

\section{Introduction}

The vibro-acoustic characteristics of panel-like structures have been extensively investigated in the past decades due to the significance of numerous industrial structures that are modelled, to a first approximation, as finite flat panels. Typical examples include high speed trains, automobiles, ship hulls, etc. An extensive literature is available either on the vibro-acoustics of single panel structures [1-2] or on panel-like structures consisting of certain kinds of attached components, e.g. rib-stiffened panel structures [3], double plates with typical stiffeners [4], framed panel structures [5], etc.

The presence of local masses attached to the surfaces of structures, which affect the structural vibro-acoustics, has been widely studied based on conventional modelling 
A Study on the Effects of Local Added Masses on the Natural and the Sound Radiation Characteristics of Thin Plate Structures

techniques, e.g. the analytical, numerical-, and experimental methods, and/or any appropriate combination of two or more of them For example, a mathematical model describing the lateral free vibration of a clamped-clamped cracked beam carrying concentrated masses has been derived by Alsaid [6]. The local mass effects on the vibration of panel structures have been investigated by Kopmaz [7] and Wong [8], while the local mass effects on panel radiation characteristics have been investigated by $\mathrm{Li}$ [9] and Sharma [10], mainly based on the analytical and the numerical modelling. Further relevant investigations include the local mass effects on vibro-acoustic characteristics of cylindrical shells [11] and a honeycomb panel [12] based on the numerical and the experimental modelling. All these investigations have clearly shown that the modification level of the panel vibro-acoustic characteristics depends greatly on both the location and the size of the attached mass and its distribution over the panel surfaces. However, there were no direct comparisons showing how the degree of influence on the modal vibration of a panel may differ from that on its modal radiation when the panel structure is subjected to the same level of point mass loading.

It has been well understood that the flexible modes of a thin panel structure behave quite differently in terms of vibration and radiation [13]. It is typically exhibited that the structural modes in general do not radiate independently so that a portion of the radiated power is attributable to the acoustic interaction between vibrating modes. Consequently, it is very important to be able to distinguish the local mass effects on the modal vibration characteristics of an elastic panel from those on the modal radiation characteristics so that more efficient passive techniques can be further generated for the noise and the vibration control [14], especially for the low and medium frequency ranges.

With the above in mind, a simple mass-plate model is set up in the present study, as shown in Fig. 1, in order to gain a deep insight into the mass effects on the vibro-acoustics of the plate flexible modes. An analytical mode decomposition method is adopted on the basis of simply supported rectangular plates, which allows the calculations of both the vibrational modes of the panel (including the rib-stiffened panel cases in a parallel investigation) and the associated acoustic radiation of the structures in a baffled condition. The modified modal vibration and radiation characteristics (e.g. the natural frequencies and corresponding modal radiation coefficients) of the plate after the attachment of the point mass are then directly compared with those of the "unloaded" plate modes. Following the evidence by the analytical modelling, the corresponding phenomena are illustrated with numerical simulations for other boundary condition cases of the plate.

In the text that follows, an analytical method used for finding the eigenfrequencies of a rectangular plate with a point mass attachment is presented in Section 2. In Section 3, the vibration response of a plate subjected to a harmonic point excitation at the local mass is presented in terms of mode summation and the corresponding sound power, which is presented in terms of a discretized surface approach, with the self-radiation and mutualradiation being addressed. Then, in Section 4, both the analytical and the numerical study are conducted to assess the contribution of individual modes to the total vibration energy response and to the radiated sound power of the rectangular plate. Thus, the physical mechanisms are interpreted in an explicit way. The significance of the local mass impact on the modal vibro-acoustic characteristics of plates is finally given in Section 5.

\section{Eigenfrequencies of a rectangular plate with a point mass attachment}

In this section, an analytical method is employed to find the eigenfrequencies of a rectangular plate with a point mass attachment.

Consider a thin plate with a local mass located at the point $\left(x_{\mathrm{C}}, y_{\mathrm{C}}\right)$. The width, length, and thickness of the plate are $a, b$ and $h$, respectively, as shown in Fig. 1. 


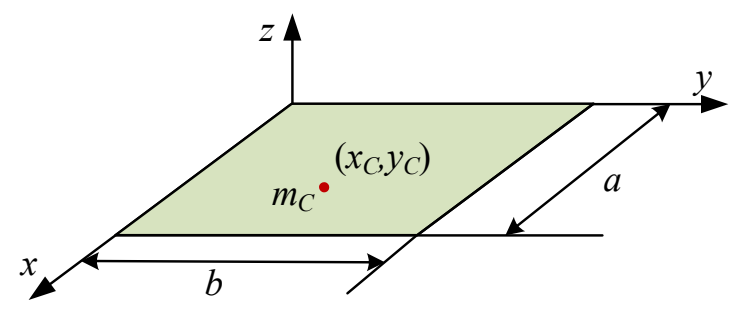

Fig. 1 Arectangular plate with a local mass

By neglecting the effects of shear deformation and rotary inertia, the governing equation of a uniform isotropic rectangular plate carrying a point mass may be written as

$$
D \nabla^{4} w(x, y, t)+\rho h \ddot{w}(x, y, t)=-m_{\mathrm{C}} \delta\left(x-x_{\mathrm{C}}, y-y_{\mathrm{C}}\right) \ddot{w}(x, y, t)
$$

where, $w(x, y, t)$ is the displacement at a general location $(x, y), D=E h^{3} /\left[12\left(1-v^{2}\right)\right]$ is the flexural rigidity, $E$ and $v$ are Young's modulus and Poisson's ratio, respectively, $\rho$ is the density of the plate, $m_{\mathrm{C}}$ is the weight of the local mass, and $\delta(x, y)$ is the Dirac delta function.

The vibration response at a grid of points $(x, y)$ on the surface of the plate obtained by using the modal summation method is given as

$$
w(x, y, t)=\sum_{k} q_{k}(t) \varphi_{k}(x, y)
$$

where, $q_{k}(t)$ are the modal coordinates, $\varphi_{k}(x, y)$ is the $k$ th mode shape function of the structure at the point $(x, y)$, respectively, which satisfies the natural boundary conditions of the plate. The orthogonality property of $\varphi_{k}(x, y)$, if mass normalized, gives

$$
\begin{aligned}
& \int_{V} \rho h \varphi_{k}(x, y) \varphi_{k^{\prime}}(x, y) \mathrm{d} x=\left\{\begin{array}{l}
1, k=k^{\prime} \\
0, k \neq k^{\prime}
\end{array}\right. \\
& \int_{V} D \nabla^{2} \varphi_{k}(x, y) \varphi_{k^{\prime}}(x, y) \mathrm{d} x=\left\{\begin{array}{r}
\omega_{k}^{2}, k=k^{\prime} \\
0, k \neq k^{\prime}
\end{array}\right.
\end{aligned}
$$

Assuming that only $K$ modes are involved in the calculation, one can rewrite the governing equation in matrix form by combining Eq. (1)-(3)

$$
\left[\boldsymbol{\Lambda}-\omega^{2}\left(\mathbf{E}+m_{c} \mathbf{\Phi}_{c}\right)\right] \mathbf{q}=\mathbf{0}
$$

In the above equation, $\boldsymbol{\Lambda}=\operatorname{diag}\left(\omega_{1}^{2}, \omega_{2}^{2}, \cdots \omega_{K}^{2}\right)$ is the $(K \times K)$ diagonal matrix and $\omega_{k}$ is represented to the $k$ th natural frequency of the unloaded plate, $\mathbf{E}$ is the $(K \times K)$ unit matrix, $\boldsymbol{\Phi}_{c}$ is the $(K \times K)$ matrix, whose $\left(k, k^{\prime}\right)$ th element is given by

$$
\Phi_{c, k k^{\prime}}=\iint_{S} \varphi_{k}(x, y) \varphi_{k^{\prime}}(x, y) \delta\left(x-x_{c}, y-y_{c}\right) \mathrm{d} x \mathrm{~d} y
$$

Equation (4) represents the eigenvalue equation, which can be solved by an eigenvalue solver to obtain natural frequencies and the corresponding modes. 
For example, a rectangular, thin plate structure is loaded by a mass $m_{\mathrm{C}}$ at the location $\left(x_{\mathrm{C}}, y_{\mathrm{C}}\right)$. If simply supported boundaries are assumed, the plate mode $\varphi_{k}(x, y)$ can now be separated into $x$ and $y$ directions, i.e. $\varphi_{k}(x, y)=\phi_{m}(x) \psi_{n}(y)$. The mass-normalized mode shape and the corresponding natural frequency in this case can be written as

$$
\begin{aligned}
& \varphi_{k}(x, y)=\frac{2}{\sqrt{\rho a b h}} \sin \left(\frac{m \pi x}{a}\right) \sin \left(\frac{n \pi y}{b}\right) \\
& \omega_{k}=\sqrt{\frac{D}{\rho h}}\left[\left(\frac{m \pi}{a}\right)^{2}+\left(\frac{n \pi}{b}\right)^{2}\right]
\end{aligned}
$$

where, $D$ is the dynamic stiffness of the plate, and $\rho$ and $h$ are the mass density and the thickness of the plate, respectively. Combining Eq. (5) and (6) with Eq. (7), one can obtain the natural frequencies and mode shapes of the loaded plate.

$$
\left|\boldsymbol{\Lambda}-\omega^{2}\left(\mathbf{E}+m_{c} \mathbf{\Phi}_{c}\right)\right|=0
$$

Equations (4)-(5) imply that (1) the eigenfrequency of the mass-plate system is related to the plate mode shapes at the point mass location $\left(x_{c}, y_{c}\right)$; $(2)$ when the point mass is located at the node of vibration mode in the $x$ or $y$ directions, neither the natural frequency nor the modal shape in this particular mode can be affected, e.g. if a simply supported plate carries a point mass located at its centre, according to Eq. (6), $\varphi_{2}\left(x_{c}, y_{c}\right)=0$. Consequently, according to Eq. (5) and (7), $\omega_{c, 2}=\omega_{2}$, i.e., the mass loading has a negligible effect on the 2nd order natural frequency and mode shape of the plate.

\section{Vibration and radiation responses of the plate subjected to a harmonic force point excitation}

In this section, the mass-plate system vibration response to harmonic point excitation is presented using the modal summation method, and the associated sound power, both the selfradiation and the mutual-radiation, is illuminated in terms of discretized surface approach.

\subsection{Vibration response}

Assume a time harmonic force at the point $\left(x_{0}, y_{0}\right)$ based on the free vibration mode described in Section 2. According to Eq. (1) and Eq. (5), the mass-plate system vibration response may be written, in matrix form, as

$$
\left[\boldsymbol{\Lambda}-\omega^{2}\left(\mathbf{E}+m_{c} \mathbf{\Phi}_{c}\right)\right] \mathbf{q}=F_{0} \boldsymbol{\varphi}_{\mathbf{0}}^{\mathrm{T}}
$$

where, $F_{0}$ is the harmonic force and $\boldsymbol{\varphi}_{0}$ is the $(1 \times p)$ modal shape vector with matrix elements $\varphi_{k}\left(x_{0}, y_{0}\right)$. The modal coordinate vector $\mathbf{q}$ of the mass-plate system can be found by solving the equation below

$$
\mathbf{q}=F_{0}\left[\boldsymbol{\Lambda}-\omega^{2}\left(\mathbf{E}+m_{c} \mathbf{\Phi}_{c}\right)\right]^{-1} \boldsymbol{\varphi}_{\mathbf{0}}^{\mathrm{T}}
$$

The mass-plate harmonic displacement response at an arbitrary point $(x, y)$, obtained by the modal summation method, can then be expressed as 

the Natural and the Sound Radiation Characteristics of Thin Plate Structures

$$
w(x, y, t)=F_{0} \boldsymbol{\varphi}\left(\left[\boldsymbol{\Lambda}-\omega^{2}\left(\mathbf{E}+m_{c} \boldsymbol{\Phi}_{c}\right)\right]^{-1} \boldsymbol{\varphi}_{\mathbf{0}}^{\mathrm{T}}\right)
$$

where, $\boldsymbol{\varphi}$ is the vector formed by the modal shape values (i.e. eigenvectors of $\left.\left|\boldsymbol{\Lambda}-\omega^{2}\left(\mathbf{E}+m_{c} \mathbf{\Phi}_{c}\right)\right|=0\right)$ at the $(x, y)$ location of the plate. In the case of small mass loading, the mode shapes of the plate tend to be affected fairly little.

As for harmonic vibrations, the time-average kinetic energy of the plate is computed from

$$
K=\frac{1}{2} \int_{S}\left[\frac{1}{T} \int_{0}^{T} \rho h|v(x, y, t)|^{2} \mathrm{~d} t\right] \mathrm{d} S
$$

where, $S$ is the area of the vibration surface and $T$ is the time over one period, where $v(x, y, t)$ is the velocity response of the plate at point $(x, y)$. In general, the expression of kinetic energy given in Eq. (11) can be used to well represent the vibration level of a plate surface.

Substituting the modal summation method from Eq. (10) into Eq. (11), the time-average kinetic energy can be determined by using a matrix form as

$$
K=\frac{\rho h}{4} \int_{0}^{a} \int_{0}^{b} \mathbf{q}_{\mathbf{v}}{ }^{\mathrm{H}} \boldsymbol{\varphi}^{\mathrm{T}} \boldsymbol{\varphi} \mathbf{q}_{\mathbf{v}} \mathrm{d} x \mathrm{~d} y
$$

where, the two superscripts $\mathrm{H}$ and $\mathrm{T}$ denote the complex conjugate and the vector transpose, respectively, and $\mathbf{q}_{\mathbf{v}}$ is the $(p \times 1)$ complex modal vector of velocities, which can be found by taking the time derivative of Eq. (10).

\subsection{Radiation response}

The total acoustic power radiated from the plate can be evaluated by introducing the socalled "power transfer matrix" [15] and the "radiation resistance matrix" [3] and by presenting the discretized surface approach based on elementary radiators.

Divide the baffled plate into a grid of $R$ rectangular elements, and assume that the size of each radiator is much smaller than the acoustic wavelength of the structure. The total sound power is given by

$$
P=\mathbf{v}^{\mathrm{H}} \boldsymbol{\Psi}^{\mathrm{T}} \mathbf{R} \boldsymbol{\Psi} \mathbf{v}=\mathbf{v}^{\mathrm{H}} \mathbf{A} \mathbf{v}
$$

In Eq. (13), $\boldsymbol{\Psi}$ is the $(R \times K)$ matrix formed by the exact modal shape vectors $\left\{\boldsymbol{\varphi}_{1}, \boldsymbol{\varphi}_{2} \cdots \boldsymbol{\varphi}_{K}\right\}^{\mathrm{T}}, \mathbf{v}$ is the $(K \times 1)$ associated complex modal vector of velocities, and $\mathbf{R}$ is the $(R \times R)$ matrix given by

$$
\mathbf{R}=\frac{\omega^{2} \rho_{0} S_{e}^{2}}{4 \pi c_{0}}\left[\begin{array}{cccc}
1 & \frac{\sin \left(k R_{12}\right)}{k R_{12}} & \cdots & \frac{\sin \left(k R_{1 R}\right)}{k R_{1 R}} \\
\frac{\sin \left(k R_{21}\right)}{k R_{21}} & 1 & \cdots & \frac{\sin \left(k R_{2 R}\right)}{k R_{2 R}} \\
\vdots & \vdots & \ddots & \vdots \\
\frac{\sin \left(k R_{R 1}\right)}{k R_{R 1}} & \frac{\sin \left(k R_{R 2}\right)}{k R_{R 2}} & \cdots & 1
\end{array}\right]
$$


Equation (14) is the so-called "radiation resistance matrix", where $\rho_{0}$ is the density of the air, $c_{0}$ is the sound speed in the air, $S_{e}$ is the area of each radiator, and $R_{i j}$ is the distance between the centres of the $i$ th and $j$ th elements.

From Eq. (13) and Eq. (14), $\mathbf{A}$ is the $(K \times K)$ matrix determined by

$$
\mathbf{A}=\boldsymbol{\Psi}^{\mathrm{T}} \mathbf{R} \boldsymbol{\Psi}
$$

A is referred to as "the power transfer matrix". The main diagonal terms of A represent the sound power radiated directly from each individual vibrating mode, i.e. the so-called "self-radiation" parts, while the off-diagonal terms account for the sound power radiated via the acoustic coupling between different orders of vibrating modes, i.e. the so-called "mutualradiation" parts [13]. Consequently, the self-radiated sound power can be obtained from Eq. (13) by letting the off-diagonal terms of $\mathbf{A}$ be zero, while the rest of the total power radiation accounts for the mutual-radiation part.

\subsection{Radiation efficiency}

The radiation efficiency of a vibrating structure is generally defined as

$$
\sigma=\frac{P}{\rho_{0} c_{0} S\left\langle\overline{v^{2}}\right\rangle}
$$

where, $P$ is the total radiated sound power calculated by Eq. (13), and $\left\langle\overline{v^{2}}\right\rangle$ is the spaceaverage mean square velocity of the plate surface vibration. Clearly, $\left\langle\overline{v^{2}}\right\rangle$ is related to the time-average kinetic energy by

$$
K=\frac{M_{P}}{2}\left\langle\overline{v^{2}}\right\rangle
$$

The modal radiation efficiency of a particular vibrating mode can then be calculated in a similar way as in Eq. (16) but with $P$ and $\left\langle\overline{v^{2}}\right\rangle$ to be replaced by the vibro-acoustic responses associated only with that particular vibrating mode.

\section{Results and discussion}

In this section, a set of mass-plate numerical examples are set up to investigate the local mass effects on the modal vibro-acoustic characteristics of thin, rectangular plate structures. Extra effort is devoted to highlight different effects on the plate modal vibration and the associated modal radiation.

\subsection{Model description}

The mass-plate models employed in the investigation are briefly introduced in this subsection. The material properties and dimensions of the plate are listed in Table 1. The local mass effects on the vibro-acoustic characteristics of the plate are investigated by varying the mass value of the point mass (in percentage of the total plate mass) and the connecting 
A Study on the Effects of Local Added Masses on

the Natural and the Sound Radiation Characteristics of

Thin Plate Structures

location with the plate surface. Three mass values and three connecting locations are employed as shown in Table 2.

Table 1 Material properties and dimensions of the plate

\begin{tabular}{|c|c|c|c|c|c|}
\hline $\begin{array}{c}\text { Length, } \\
\mathrm{mm}\end{array}$ & $\begin{array}{c}\text { Width, } \\
\mathrm{mm}\end{array}$ & $\begin{array}{c}\text { Thickness, } \\
\mathrm{mm}\end{array}$ & $\begin{array}{c}\text { Young's } \\
\text { modulus, } \mathrm{GPa}\end{array}$ & $\begin{array}{c}\text { Density, } \\
\mathrm{kg} \cdot \mathrm{m}^{-3}\end{array}$ & $\begin{array}{c}\text { Poisson's } \\
\text { ratio }\end{array}$ \\
\hline 790 & 640 & 1.5 & 210 & 7850 & 0.3 \\
\hline
\end{tabular}

Table 2 Point masses employed

\begin{tabular}{|c|c|c|c|}
\hline & Mass 1 & Mass 2 & Mass 3 \\
\hline$\left(m_{C} / M_{P}\right)$ & $6 \%$ & $15 \%$ & $30 \%$ \\
\hline & Point 1 & Point 2 & Point 3 \\
\hline$\left(x_{C} / a, y_{C} / b\right)$ & $(0.437,0.585)$ & $(0.5,0.5)$ & $(0.17,0.273)$ \\
\hline
\end{tabular}

The plate is first assumed to be with four edges simply supported so that a physical interpretation can be made in an efficient and analytical way. Then, the plate boundaries are assumed to be fully fixed so as to simulate a condition more closely related to the practical engineering.

In the numerical examples below, all the calculations for simply supported plates are done by analytical equations derived in Sections 2-3, e.g. the natural frequencies from Eq. (5)-(7), the total radiated sound power from Eq. (13)-(14). Note that the exact plate mode shapes after mass loading are determined by the eigenvectors of Eq. (4). However, when calculating the radiation, unloaded plate mode shapes are used in Eq. (15) for simplicity due to the minor influence of the loaded masses in these specified cases. For example, representative mode shapes of the plate before and after mass loading are compared in Fig B1 and Fig B2 in Appendix B. The comparison clearly shows that the mass effects on mode shapes are indeed fairly modest.

On the other hand, FE simulations of simply supported mass-plate systems conducted by ANSYS are used for simulation validity in which quadrilateral meshes are generated with element size of $0.02 \mathrm{~m}$, which is less than one-sixth of the plate wavelength at $200 \mathrm{~Hz}$. The analytical and the FE simulation results are given in Table A1 and Table A2, respectively, of Appendix A. A comparison between the two sets of results shows that the analytical method results are in agreement with the FE-simulation results, though FE-simulation results exhibit lower values than the former. Finally, FE models of fully fixed mass-plate systems are established in Subsection 4.2 and are used for verifying the mass effects on natural frequencies of the plate, which are listed in Table A3 of Appendix A.

\subsection{Natural frequencies}

The first 15 orders of natural frequencies of the mass-plate systems for the following 4 different mass-plate coupling cases are calculated as shown in Fig. 2-5, and the comparisons between Cases 1 and 2 and those between Cases 3 and 4 are given in Tables A1 and A3, respectively.

Table 3 Cases of mass-plate systems

\begin{tabular}{|c|c|c|c|}
\hline No. & Boundary Condition & Mass Value & Mass Location \\
\hline Case 1 & Simply supported & Mass-1, -2 , and -3 & Point-1 only \\
\hline Case 2 & Simply supported & Mass- 1 only & Point-1, -2 and -3 \\
\hline Case 3 & Fully constrained & Mass-1, -2 , and -3 & Point-1 only \\
\hline Case 4 & Fully constrained & Mass- 1 only & Point $-1,-2$, and -3 \\
\hline
\end{tabular}


Generally speaking, the natural frequencies of the plate structures tend to be reduced after adding a point mass, as can be seen from Tables A1-A3 in Appendix A. Here, the decreasing levels of natural frequencies from Fig. 2-5 are compared in percentages for the above four cases.

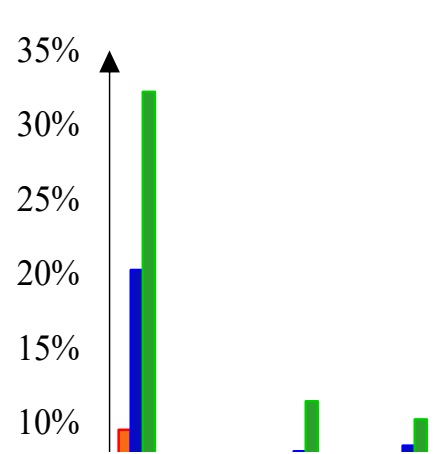

Fig. 2 Decrease in natural frequencies, Case 1

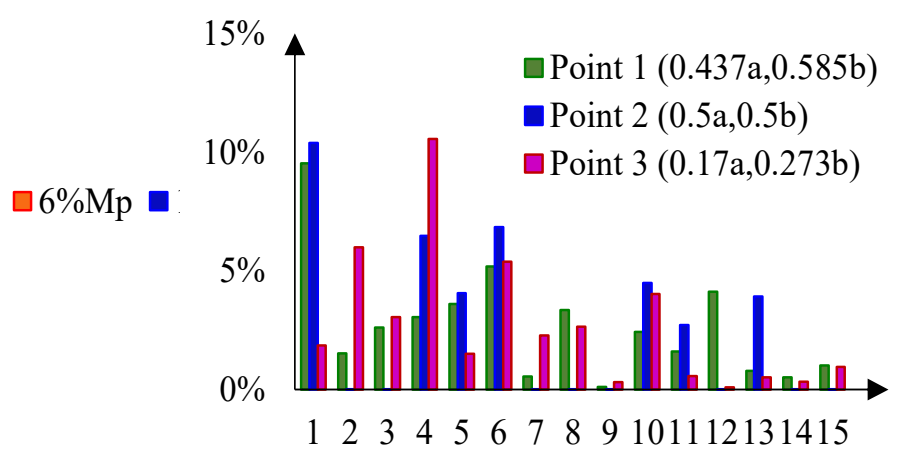

Fig. 3 Decrease in natural frequencies, Case 2

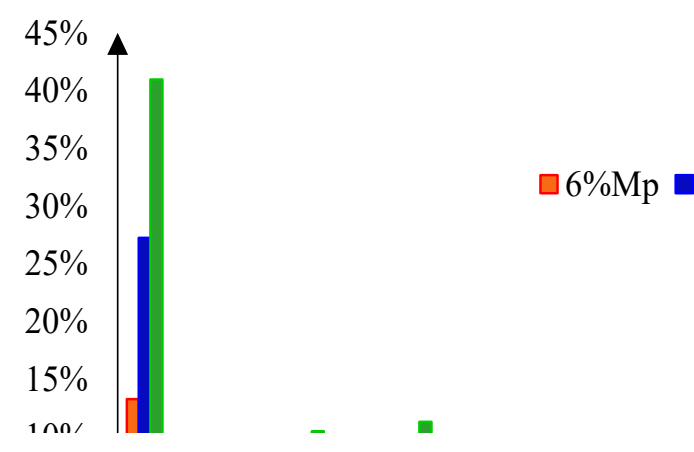

Fig. 4 Decrease in natural frequencies, Case 3

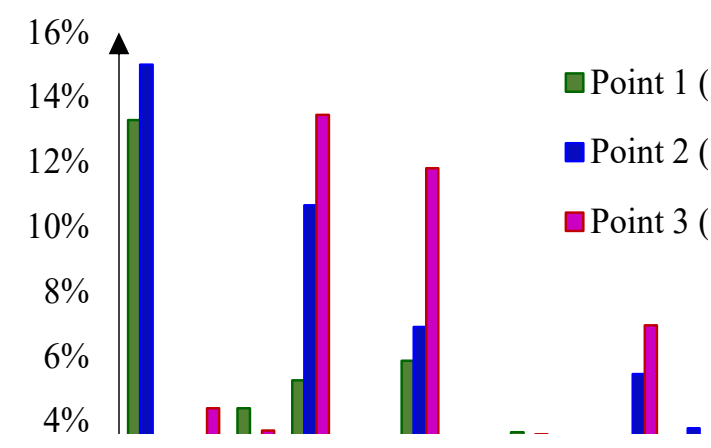

Fig. 5 Decrease in natural frequencies, Case 4

From Fig. 2 and Fig. 4 one can see that, for a fixed interface location, the following is valid: the larger the local mass is, the bigger is the dynamic influence on the plate modes, especially with the lower order modes, which tend to be more affected than the higher order ones. The above phenomena occur regardless of the boundary conditions of the plate structures. However, Fig. 3 and Fig. 5 show that, in the case of the same point mass but with various interface locations, the trends in the impact on the plate modes become fairly complicated in that the higher order modes may be much more significantly affected than the lower order ones. Meanwhile, the degrees of influence on different orders of plate modes also depend on the detailed boundary conditions.

Therefore, one may conclude that, by a proper combination of the local mass value, the coupling location, and the plate boundary settings, it is possible to achieve an efficient passive control for a particular mode of the plate.

\subsection{Vibration response}

Figures 6 and 7 compare vibrational energy responses of the plate before and after being connected with a point mass, corresponding to the mass-plate coupling, for Cases 1- and 2, respectively.

It can be seen that the surface velocity response of the plate structure at the first natural frequency is most significantly affected after the local mass has been attached.

The results suggest that if the plate vibration is dominated by its lower order modes, the mass value effects tend to be much more significant than the coupling location effects. 

the Natural and the Sound Radiation Characteristics of Thin Plate Structures

However, if the plate vibration is dominated by the higher order modes, the influence of the mass values and that of the coupling locations tend to be similar.

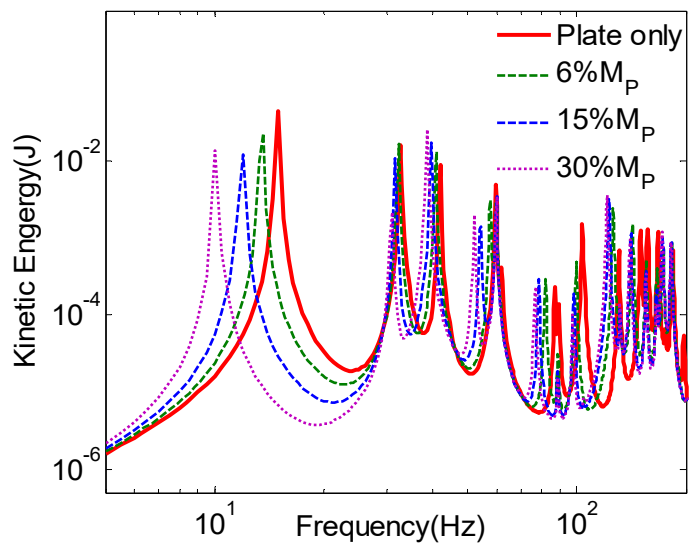

Fig. 6 Influences on the kinetic energies of a plate with a varied point mass (Case 1)

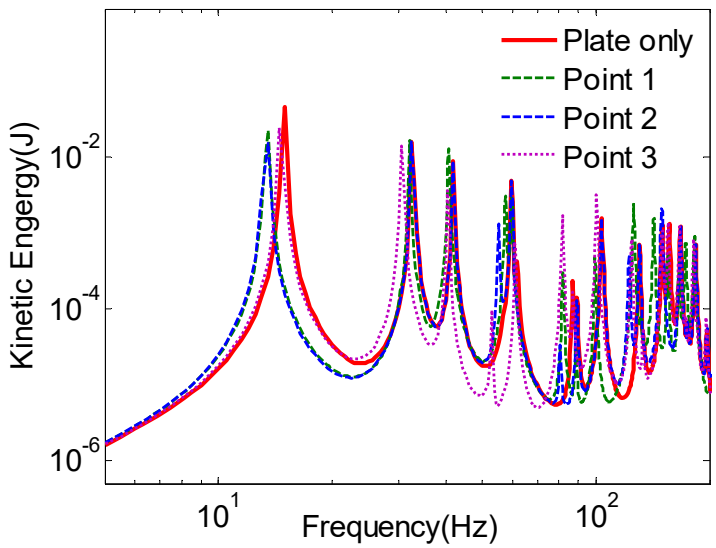

Fig. 7 Influences on the kinetic energies of a plate with a varied connecting location mass (Case 2)

\subsection{Sound power radiation}

In addition to the comparisons of vibration shown in Fig. 6 and 7, comparisons of the sound power radiation from the plate in the Case 1 and Case 2 are given in Fig. 8 and 9, respectively.

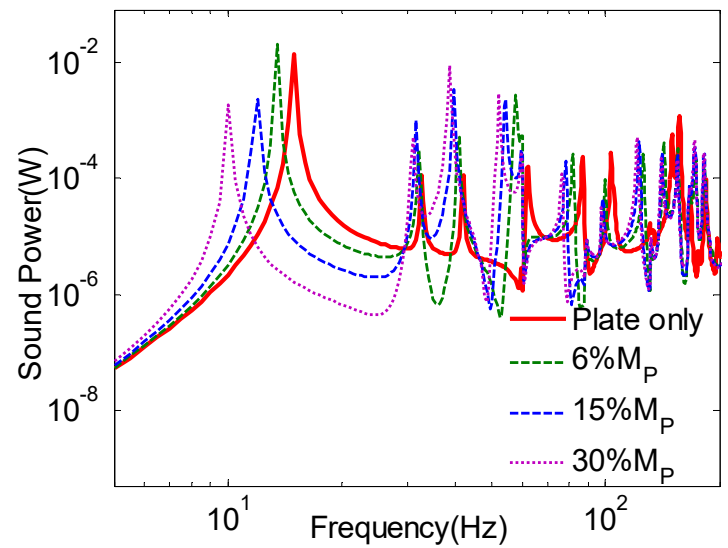

Fig. 8 Influences on the sound power of a plate with a varied point mass (Case 1)

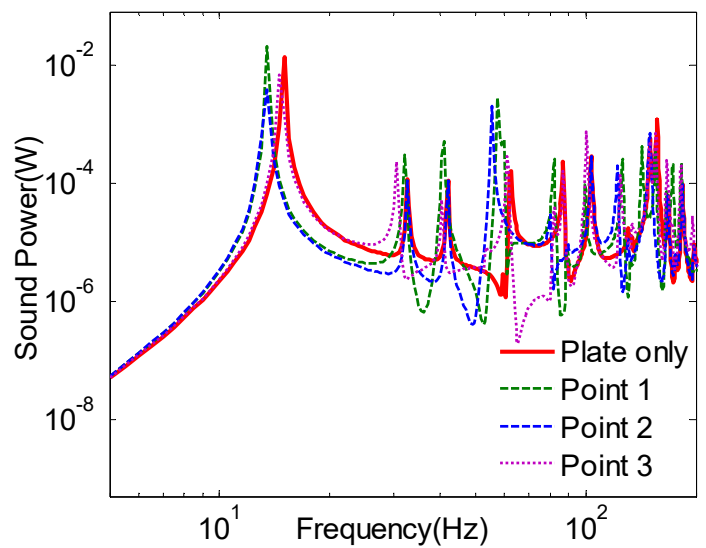

Fig. 9 Influences on the sound power of a plate with a varied connecting location mass (Case 2)

It can be seen that, at the fundamental frequency of the plate, the local mass effects on the acoustic radiation of the plate are very similar to those on the plate vibration; thus, a point mass tends to reduce both the vibration and the radiation at the fundamental frequency. However, if the radiation is mainly from the high order vibrating modes, the effects of point mass on the radiation become quite different from those on the vibration. One can see that an added mass can significantly increase the acoustic radiation from the higher order modes. These phenomena can be noted for both the mass variation and coupling point variation cases.

To understand the reasons why the radiation from the higher order modes has been increased by an added mass, the modal radiation efficiencies for the first 3 bending modes of the plate when different point masses are attached to the plate vibrating surfaces are shown and compared in Figures 10-13. 


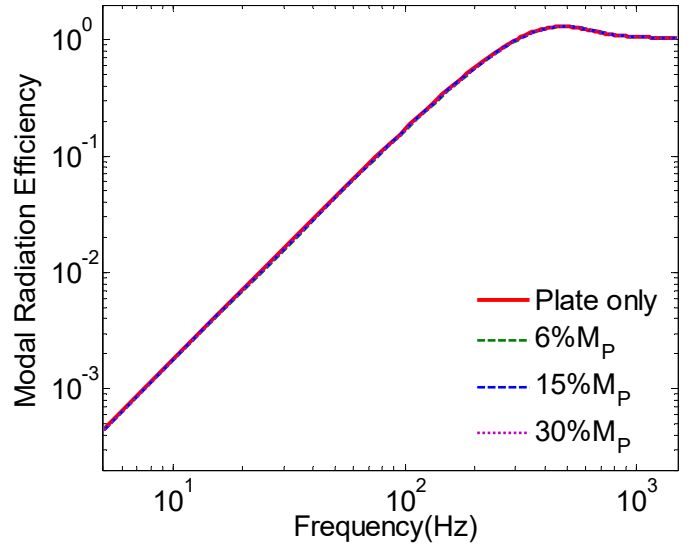

Fig. 10 Influences on the 1st radiation efficiencies of a plate with a varied point mass (Case 1)

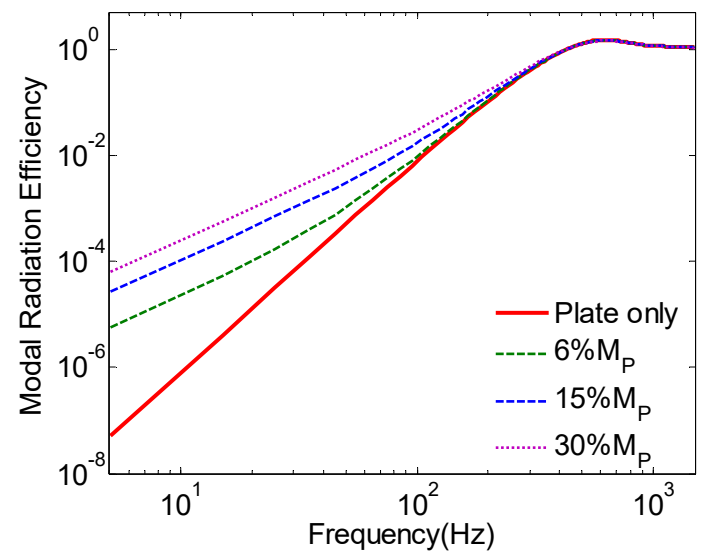

Fig. 12 Influences on the 2 nd radiation efficiencies of a plate with a varied point mass (Case 1)

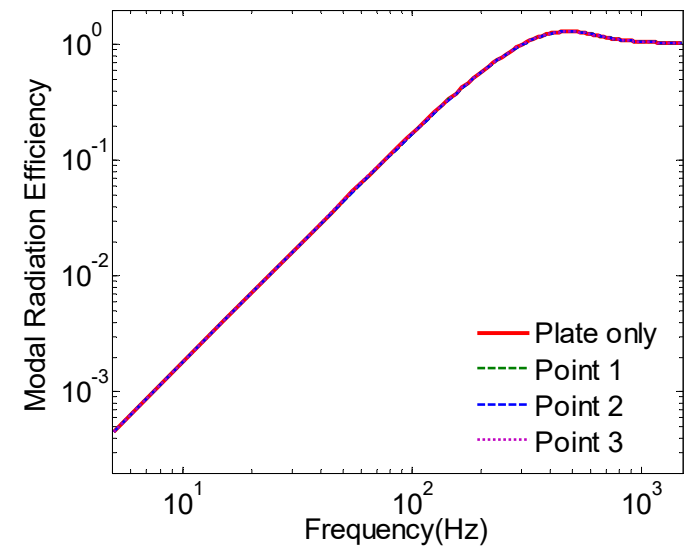

Fig. 11 Influences on the 1st radiation efficiencies of a varied connecting location mass (Case 2)

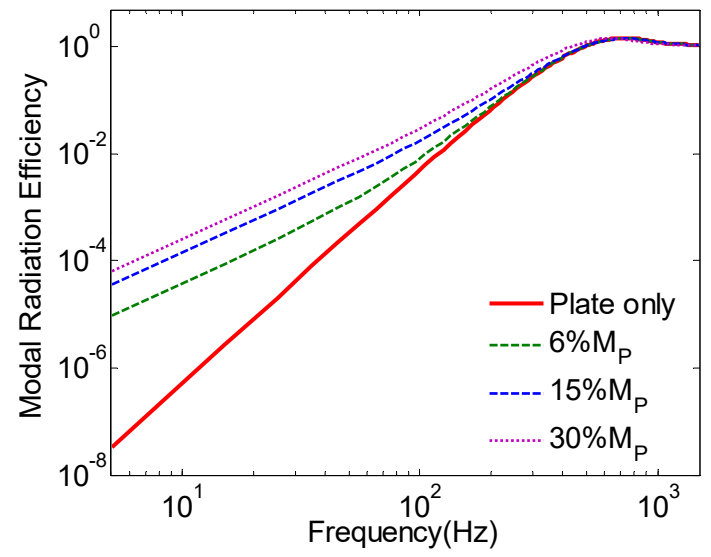

Fig. 13 Influences on the 3 rd radiation efficiencies of a plate with a varied point mass (Case 1)

Figures 10-11 show that the radiation efficiency of the fundamental mode is almost not affected by an added mass, regardless of the exact point mass value or its varied connecting location. However, the radiation efficiencies for the second (Fig. 12) and the third (Fig. 13) bending mode of the plate have all been increased, while the larger the added mass value is, the sharper is the increase in the radiation efficiency.

Figures 10-13 can well explain why the acoustic radiation from the plate is s increased in the region dominated by the higher order modes after the attachment of a point mass (e.g. in Fig. 8-9).

\subsection{Self-radiation and mutual-radiation}

Finally, local mass effects on the self-radiation and mutual- radiation characteristics of plate vibrating modes are investigated analytically.

Figures 14-15 show the self-radiation and mutual-radiation versus the total sound power radiation before and after a point mass is attached to the plate, respectively.

One can see that an added local mass can significantly increase the mutual- radiation of the plate bending modes, especially for the higher order vibrating modes. This clearly indicates that the acoustic interactions between different vibrating modes can be significantly increased by the local point mass. 


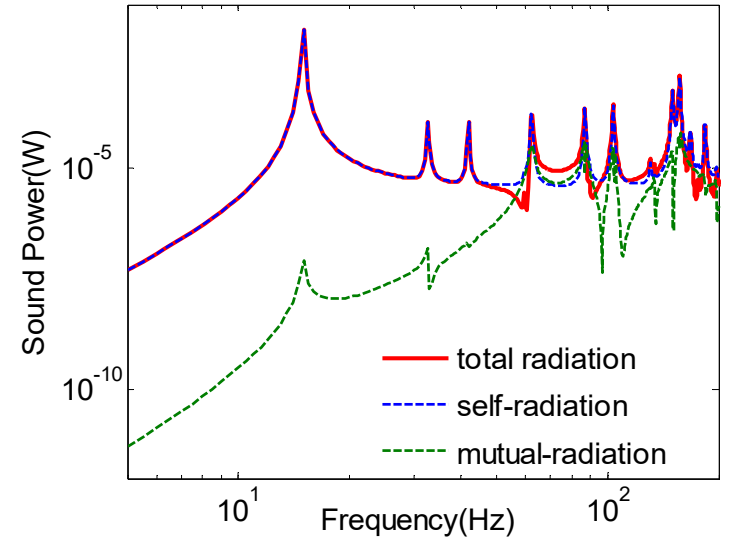

Fig. 14 Total, self- and mutual-radiation sound power of a simply supported plate

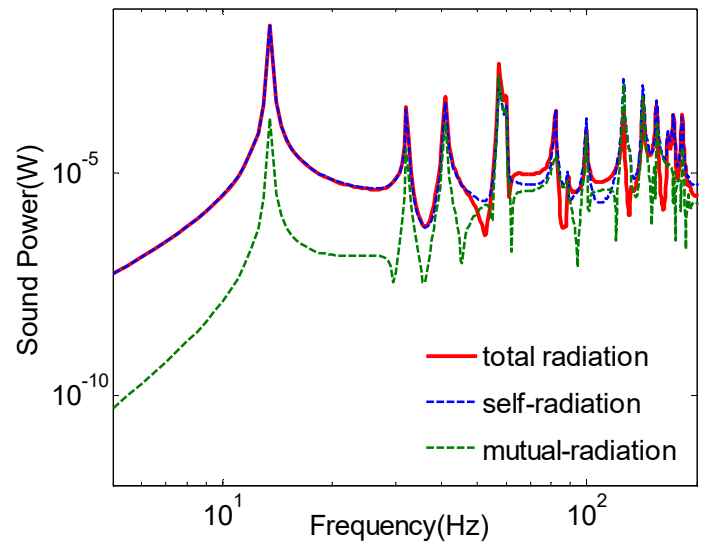

Fig. 15 Total, self-and mutual-radiation sound power of a mass-plate (Point 1)

\section{Conclusions}

The present study was developed to highlight the differences among local mass effects on the vibration and the radiation behaviour of flexible modes of the flat panel structures. A simple model comprising a local mass attached to a rectangular plate surface is set up, which allows us a deep insight into how a local mass can affect the inherent modal characteristics, and the corresponding vibration and radiation characteristics of a generic, thin, flat panel structure. The influential phenomena are first investigated analytically and then verified with FE-numerical simulations. The following conclusions can be drawn from the investigations.

(1) The modal dynamics of the lower order plate modes tend to be more affected by a local mass than that of the higher order ones if the attachment location of the mass to the plate is fixed. However, such influential trends can be significantly altered when varying the location of the same local mass attachment to the plate: a higher order mode can be more significantly affected than a lower order mode provided that a mass-plate coupling location is properly chosen.

(2) When the plate vibration is dominated by its lower order modes, the mass value effects tend to be much more significant than the coupling location effects. However, if the plate vibration is dominated by the higher order modes, the influence of the mass values and those of the coupling locations tend to be similar.

(3) In the low frequency range, e.g. close to the fundamental frequency of the plate, a point mass tends to reduce both the vibration and the radiation at the fundamental frequency. However, at higher frequencies, where the radiation is mainly from the high-order vibrating modes, an added mass can significantly increase the acoustic radiation from the higher order modes. This is because the radiation efficiency of the fundamental mode is almost not affected by an added mass, while the radiation efficiencies of the higher order vibrating modes can be significantly increased by the added local mass.

(4) An added local mass can significantly increase the mutual-radiations of the plate bending modes, especially those for the higher order vibrating modes. This suggests that the acoustic interactions between different vibrating modes can be significantly increased by the local point mass. 


\section{Appendix A: Natural frequencies of the plate before and after the attachment of a point mass}

Natural frequencies of the mass-plate system of 4 different coupling cases described in Subsection 4.2 are calculated and compared with the natural frequencies of the single plate case. On the other hand, the natural frequencies obtained by the analytical method and those by FE simulations have been compared. Note that, in the calculation below, only the point mass value is varied in Case 1 and Case 3, while only the point mass location is varied in Case 2 and Case 4.

Table A1 Analytical results of natural frequencies of simply supported plates (Case 1 vs. Case 2)

\begin{tabular}{|c|c|ccc|ccc|}
\hline \multirow{2}{*}{$\begin{array}{c}\text { Mode } \\
\text { Order }\end{array}$} & \multirow{2}{*}{$\begin{array}{c}\text { Single } \\
\text { plate }\end{array}$} & \multicolumn{3}{|c|}{ Case 1 } & \multicolumn{3}{c|}{ Case 2 } \\
\cline { 3 - 8 } & Mass 1 & Mass 2 & Mass 3 & Point 1 & Point 2 & Point 3 \\
\hline 1 & 14.9 & 13.5 & 11.9 & 10.1 & 13.5 & 13.4 & 14.6 \\
2 & 32.6 & 32.1 & 31.6 & 30.9 & 32.1 & 32.6 & 30.7 \\
3 & 41.9 & 40.8 & 39.7 & 38.8 & 40.8 & 41.9 & 40.6 \\
4 & 59.7 & 57.8 & 54.8 & 52.8 & 57.8 & 55.8 & 53.4 \\
5 & 62.2 & 59.9 & 59.8 & 59.8 & 59.9 & 59.7 & 61.2 \\
6 & 86.9 & 82.4 & 79.6 & 78.0 & 82.4 & 81.0 & 82.3 \\
7 & 89.2 & 88.7 & 88.6 & 88.6 & 88.7 & 89.2 & 87.2 \\
8 & 103.5 & 100.1 & 98.8 & 98.2 & 100.1 & 103.6 & 100.8 \\
9 & 104.7 & 104.6 & 104.5 & 104.5 & 104.6 & 104.7 & 104.3 \\
10 & 130.6 & 127.4 & 125.1 & 123.9 & 127.4 & 124.7 & 125.3 \\
11 & 134.2 & 132.1 & 131.8 & 131.7 & 132.1 & 130.6 & 133.5 \\
12 & 150.0 & 143.8 & 142.3 & 141.8 & 143.8 & 150.0 & 149.8 \\
13 & 156.7 & 155.5 & 155.3 & 155.2 & 155.5 & 150.6 & 155.9 \\
14 & 167.7 & 166.8 & 166.6 & 166.5 & 166.8 & 167.7 & 167.1 \\
15 & 175.6 & 173.8 & 173.4 & 173.2 & 173.8 & 175.6 & 173.9 \\
\hline
\end{tabular}

Table A2 FEM results of natural frequencies of simply supported plates (Case 1 vs. Case 2)

\begin{tabular}{|c|c|ccc|ccc|}
\hline \multirow{2}{*}{$\begin{array}{c}\text { Mode } \\
\text { Order }\end{array}$} & \multirow{2}{*}{$\begin{array}{c}\text { Single } \\
\text { plate }\end{array}$} & \multicolumn{3}{|c|}{ Case 1 } & \multicolumn{3}{c|}{ Case 2 } \\
\cline { 3 - 8 } 1 & 14.7 & 13.3 & 11.7 & 9.9 & 13.3 & 13.2 & 14.4 \\
2 & 32.1 & 31.6 & 31.0 & 30.3 & 31.6 & 32.1 & 30.1 \\
3 & 41.2 & 40.1 & 38.9 & 37.9 & 40.1 & 41.2 & 39.9 \\
4 & 58.6 & 56.5 & 53.1 & 50.8 & 56.5 & 54.3 & 52.0 \\
5 & 61.1 & 58.9 & 58.8 & 58.8 & 58.9 & 58.6 & 60.1 \\
6 & 85.3 & 80.2 & 76.7 & 75.0 & 80.2 & 78.8 & 80.0 \\
7 & 87.5 & 87.0 & 86.9 & 86.9 & 87.0 & 87.5 & 85.5 \\
8 & 101.5 & 97.6 & 95.9 & 95.2 & 97.6 & 101.5 & 98.5 \\
9 & 102.6 & 102.5 & 102.5 & 102.5 & 102.5 & 102.6 & 102.3 \\
10 & 127.9 & 122.9 & 119.3 & 117.6 & 122.9 & 119.1 & 121.5
\end{tabular}



the Natural and the Sound Radiation Characteristics of Thin Plate Structures

\begin{tabular}{|c|c|ccc|ccc|}
\hline \multirow{2}{*}{$\begin{array}{c}\text { Mode } \\
\text { Order }\end{array}$} & \multirow{2}{*}{$\begin{array}{c}\text { Single } \\
\text { plate }\end{array}$} & \multicolumn{3}{|c|}{ Case 1 } & \multicolumn{3}{c|}{ Case 2 } \\
\cline { 3 - 8 } & Mass 1 & Mass 2 & Mass 3 & Point 1 & Point 2 & Point 3 \\
\hline 11 & 131.4 & 129.1 & 128.8 & 128.8 & 129.1 & 127.9 & 130.6 \\
12 & 146.8 & 139.1 & 137.4 & 136.9 & 139.1 & 145.5 & 146.6 \\
13 & 153.3 & 151.9 & 151.6 & 151.6 & 151.9 & 146.8 & 152.1 \\
14 & 163.3 & 162.6 & 162.0 & 161.8 & 162.6 & 163.9 & 162.9 \\
15 & 171.5 & 168.9 & 168.1 & 167.8 & 168.9 & 171.5 & 168.9 \\
\hline
\end{tabular}

Table A3 FEM results of natural frequencies of fully constrained plates (Case 3 vs. Case 4)

\begin{tabular}{|c|c|c|c|c|c|c|c|}
\hline \multirow{2}{*}{$\begin{array}{l}\text { Mode } \\
\text { Order }\end{array}$} & \multirow{2}{*}{$\begin{array}{l}\text { Single } \\
\text { plate }\end{array}$} & \multicolumn{3}{|c|}{ Case 3} & \multicolumn{3}{|c|}{ Case 4} \\
\hline & & Mass 1 & Mass 2 & Mass 3 & Point 1 & Point 2 & Point 3 \\
\hline 1 & 27.0 & 23.4 & 19.6 & 15.9 & 23.4 & 23.0 & 26.8 \\
\hline 2 & 47.8 & 46.6 & 45.3 & 44.3 & 46.6 & 47.9 & 45.7 \\
\hline 3 & 61.6 & 58.9 & 56.8 & 55.5 & 58.9 & 61.6 & 59.3 \\
\hline 4 & 80.8 & 76.5 & 73.7 & 72.3 & 76.5 & 72.2 & 69.9 \\
\hline 5 & 81.6 & 80.9 & 80.9 & 80.9 & 80.9 & 80.8 & 81.3 \\
\hline 6 & 113.0 & 106.3 & 102.2 & 100.2 & 106.3 & 105.2 & 99.6 \\
\hline 7 & 114.4 & 113.6 & 113.5 & 113.5 & 113.6 & 113.0 & 114.3 \\
\hline 8 & 127.5 & 122.8 & 121.6 & 121.2 & 122.8 & 127.5 & 122.9 \\
\hline 9 & 132.9 & 132.4 & 132.3 & 132.2 & 132.4 & 132.9 & 131.0 \\
\hline 10 & 157.7 & 152.7 & 149.5 & 148.1 & 152.7 & 149.1 & 146.7 \\
\hline 11 & 163.9 & 161.1 & 160.5 & 160.3 & 161.1 & 157.7 & 162.1 \\
\hline 12 & 185.1 & 173.0 & 170.9 & 170.2 & 173.0 & 177.2 & 181.7 \\
\hline 13 & 185.2 & 185.2 & 185.2 & 185.2 & 185.2 & 185.1 & 185.2 \\
\hline 14 & 203.2 & 200.6 & 199.7 & 199.4 & 200.6 & 203.2 & 200.0 \\
\hline 15 & 207.5 & 205.7 & 205.5 & 205.4 & 205.7 & 207.6 & 203.2 \\
\hline
\end{tabular}




\section{Appendix B: Representative mode shapes of unloaded and mass-loaded plates}

In order to illustrate the mass effects on flexible mode shapes of the plate, representative mode shapes of the unloaded and mass-loaded plates are compared in the figures below. Mass-1, Mass-2, and Mass-3 located at Point 1 (in Table 2) are employed for the relevant FE calculations.

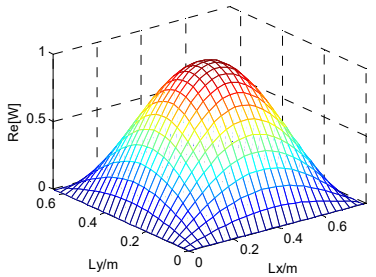

a) unloaded plate

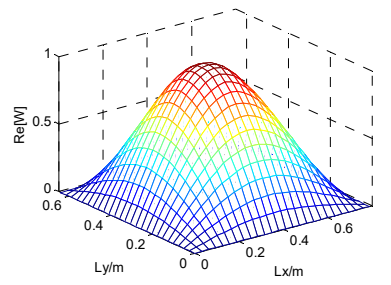

c) Mass 2, Point 1

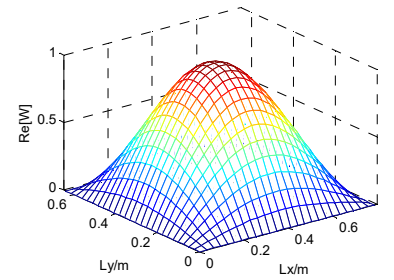

b) Mass 1, Point 1

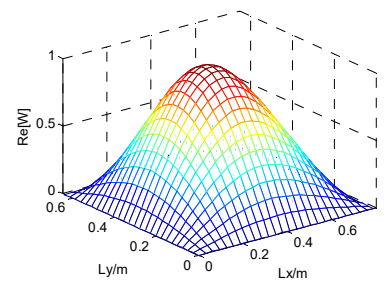

d) Mass 3, Point 1

Fig. B1 $1^{\text {st }}$ mode shapes of unloaded and mass-loaded plates

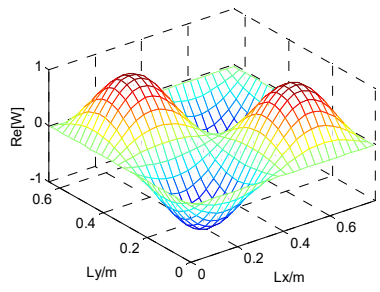

a) unloaded plate

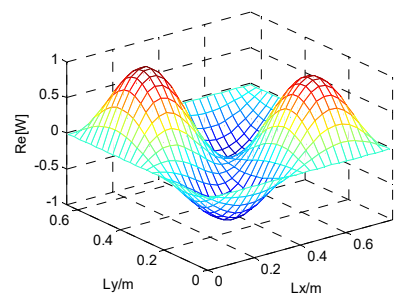

c) Mass 2, Point 1

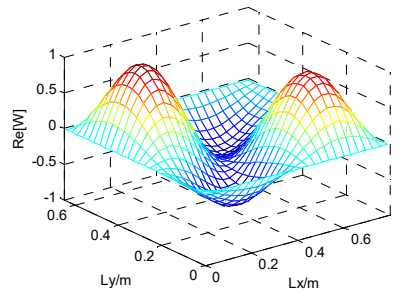

b) Mass 1, Point 1

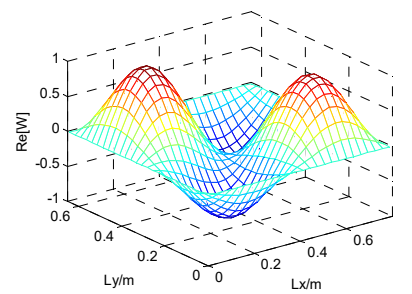

d) Mass 3, Point 1

Fig. B2 $5^{\text {th }}$ mode shapes of unloaded and mass-loaded plates 

the Natural and the Sound Radiation Characteristics of Thin Plate Structures

\section{Acknowledgements}

The authors gratefully acknowledge the financial support received from the National Natural Science Foundation of China (Nos. U1434201, and 51175300), the State Key Laboratory of Vehicle NVH and Safety Technology of China (No. NVHSKL-201503).

\section{REFERENCES}

[1] Soedel, W., 1993, Vibrations of Shells and Plates. Marcel Dekker, Inc, New York.

[2] Alshabatat, N. T., Naghshineh, K., 2015, Optimization of the Natural Frequencies of Plates Via Dimpling and Beading Techniques[J]. International Journal of Modelling \& Simulation, 32:244-254.

[3] Fahy, F.J., Gardonio, P., 2007, Sound and Structural Vibration: Radiation, Transmission and Response. Academic Press, 2nd edition.

[4] Ji, L., Sheng, X., Xiao, X., Wen, Z., Jin, X., 2015. A review of mid-frequency vibro-acoustic modelling on extruded aluminium panels for high-speed train vehicles as well as the recent developments. Journal of Modern Transportation, 23 (3): 159-168. DOI:10.1007/s40534-015-0080-4

[5] Chakraverty, S., Behera, D., 2014, Parameter Identification of Multistorey Frame Structure from Uncertain Dynamic Data[J]. Journal of Mechanical Engineering, 60 (5):331-338. DOI:10.5545/sv-jme.2014.1832

[6] Alsaid, S. A. M., Alqaisia, A. A., 2015, Influence of Crack Depth and Attached Masses on Beam Natural Frequencies[J]. International Journal of Modelling \& Simulation, 28(3):239-247.

[7] Kopmaz, O., Telli, S., 2002, Free vibrations of a rectangular plate carrying a distributed mass. Journal of Sound and Vibration, 251 (1): 39-57. DOI:10.1006/jsvi.2001.3977

[8] Wong, W.O., 2002, The effects of distributed mass loading on plate vibration behavior. Journal of Sound and Vibration, 252 (3), 577-583. DOI:10.1006/jsvi.2001.3947

[9] Li, S., Li, X., 2008, The effects of distributed masses on acoustic radiation behaviour of plates. Applied Acoustics, 69 (3): 272-279. DOI:10.1016/j.apacoust.2006.11.004

[10] Sharma, G., Sarkar, A., Ganesan, N., 2013, Acoustic directivity control by point mass attachment. 20th International Congress on Sound and Vibration (ICSV), 1: 745-752.

[11] Ekimov, A.E., Lebedev, A.V., 1996, Experimental study of local mass influence on sound radiation from a thin limited cylindrical shell. Applied Acoustics, 48 (1): 47-57. DOI:10.1016/0003-682X(95)00049-F

[12] Ando, S., Shi, Q., Nagahama, K., Saitoh, M., \& Saegusa, H., 2005, The prediction of random acoustic vibration of equipment mounted on honeycomb panel. Proceedings of the European Conference on Spacecraft Structures, Materials and Mechanical Testing, SP-581.

[13] Cunefare, K. A., 1992, Effect of Modal Interaction on Sound Radiation from Vibrating Structures, AIAA Journal, 30 (12): 2819-2828. DOI:10.2514/3.11624

[14] Beranek, L. L., Ver, I. L., 1992, Noise and vibration control engineering - Principles and applications. John Wiley \& Sons, Inc.

[15] Snyder, S.D., Tanaka, N., 1995, Calculating total acoustic power output using modal radiation efficiency. Journal of the Acoustical Society of America, 97 (3):1702-1709. DOI:10.1121/1.412048
Submitted: $\quad 06.01 .2016$
Accepted: $\quad 20.9 .2016$

Pingping Zhang

Huaiyu $\mathrm{Wu}$

Lin Ji $₫$

School of Mechanical Engineering,

Shandong University,

Jinan 250061, China

E-mail:jilin@sdu.edu.cn

Marko Jokić

Faculty of Mechanical Engineering and

Naval Architecture, University of Zagreb,

Ivana Lučića 5, 10000 Zagreb, Croatia 